
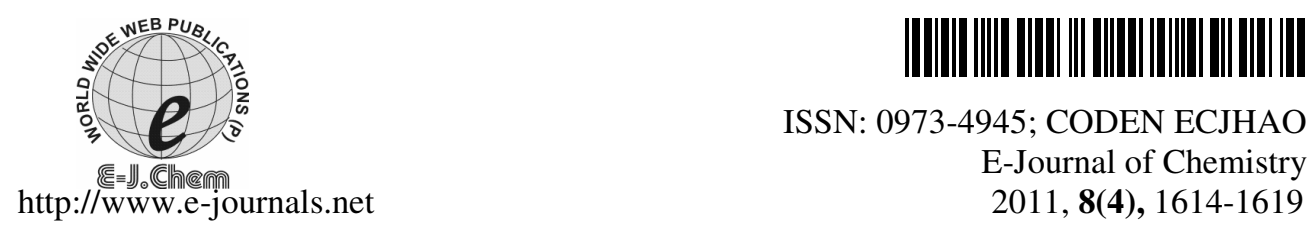

ISSN: 0973-4945; CODEN ECJHAO

E-Journal of Chemistry

2011, 8(4), 1614-1619

\title{
Comparative Study of Microwave Induced and Conventional Synthesis of Acetylated Sugar Isothiocyanates and Related Thiocarbamides
}

\author{
ATUL V. YADGIRE, GAJANAN V. KORPE and SHIRISH P. DESHMUKH* \\ P. G. Department of Chemistry \\ Shri Shivaji College, Akola-444 001 (M.S.), India \\ atulyadgire@gmail.com
}

Received 16 December 2010; Accepted 28 February 2011

\begin{abstract}
The synthesis of several acetylated sugar isothiocyanates have been carried out under microwave irradiation in excellent yields of products by using related bromides and lead thiocyanate in sodium dried xylene. Several acetylated sugar thiocarbamides have been synthesized by the interaction of respective acetylated sugar isothiocyanates with appropriate aryl amines under microwave irradiation.
\end{abstract}

Keywords: MORE, Conventional synthesis, Sugar isothiocyanates, Thiocarbamides

\section{Introduction}

Owing to present environmental awareness, attempts are being made towards the evolution of environmentally benign processes using microwave induced organic reaction enhancement (MORE). The chief features of microwave assisted reactions are the enhanced selectivity, much improved reaction rates, milder reaction conditions and formation of cleaner products. Microwave irradiation method replaces the classical one because it proves to be a clean, cheap and convenient method ${ }^{1}$. Microwave assisted synthesis affords higher yields and minor wastes. Conventional method required longer heating time, tedious procedure and the excessive use of solvents while microwave assisted reactions are often performed neat, simplifying the work-up process and minimizing the need for organic solvents. Hence, microwave method has emerged as a tool towards green chemistry. Few researchers synthesized aryl thiocarbamides ${ }^{2}$ under microwave irradiation. At the present time, microwave assisted carbohydrate chemistry is experiencing considerable growth and has potential to greatly improve the image of carbohydrate chemistry. 
Sugar isothiocyanates are the versatile reagent in the field of carbohydrate chemistry. Due to synthetic flexibility of the isothiocyanate function ${ }^{3}$, glycosyl isothiocyanates are attracting much attention among the $\mathrm{N}$-glycosides. Sugar isothiocyanates have been used for the synthesis of carbohydrate derivatives particularly having thiourea structure, of synthetic, biological and pharmaceutical interest ${ }^{4-6}$. Antiviral, antibacterial and antitumor agents have been prepared by reaction of glycosyl isothiocyanates with biologically active amines ${ }^{7,8}$.

Conventionally, in our laboratory, a number of researchers ${ }^{9-11}$ reported the synthesis of acetylated sugar isothiocyanates and related thiocarbamides. We herein report the synthesis of acetylated sugar (glucose, galactose, lactose and maltose) isothiocyanates and related thiocarbamides under microwave irradiation. In the course of our study on carbohydrate chemistry, we found that microwave irradiation could promote the acetylated sugar isothiocyanates and related thiocarbamides in moderate yields.

\section{Experimental}

All reactions were carried out in a commercially available Godrej GMC 25E 09 MRGX microwave oven having a maximum power output of $900 \mathrm{~W}$ operating at $2450 \mathrm{MHz}$. Melting points were recorded on a MAC digital melting point apparatus and are uncorrected. Thin layer chromatography was conducted on E. Merck TLC Silica gel 60 aluminium sheet.

\section{Results and Discussion}

Under microwave irradiation, acetylations of sugars (glucose, galactose, lactose and maltose) have been carried out by using appropriate amount of acetic anhydride and anhydrous sodium acetate as a catalyst. Bromination of acetylated sugars has been carried out conventionally using bromine in acetic acid and red phosphorus. Acetylated sugar isothiocyanates have been synthesized from related bromides using lead thiocyanate in sodium dried xylene under microwave irradiation. To afford products, the solvent was distilled off and sticky residue obtained was triturated with petroleum ether $\left(60-80{ }^{\circ} \mathrm{C}\right)$. The products were purified by chloroform-ether. The products were found to be desulfurized when boiled with alkaline plumbite solution.

Synthesis of tetra-O-acetyl- $\beta$-D-glucosyl isothiocyanate and tetra-O-acetyl- $\beta$ - $D$ galactosyl isothiocyanate under microwave irradiation

To a solution of tetra- $O$-acetyl- $\alpha$ - $D$-glucosyl bromide (Ia) $(0.024 \mathrm{M}, 10.0 \mathrm{~g})$ dissolved in sodium dried xylene $(25 \mathrm{~mL})$ was added lead thiocyanate $(6.0 \mathrm{~g})$. The reaction mixture was then kept under microwave irradiation for about $25 \mathrm{~min}$. at constant power P-70, the temperature of reaction mixture remain constant at $130-140{ }^{\circ} \mathrm{C}$. The resultant solution was then cooled and separated lead bromide was removed by filtration. The solvent was distilled off and sticky mass obtained as residue was triturated with petroleum ether $\left(60-80{ }^{\circ} \mathrm{C}\right)$. A pale yellow solid $(8.5 \mathrm{~g})$ was obtained. The product (IIa) was purified by chloroform-ether. m.p. $112^{\circ} \mathrm{C}$. (Scheme 1).

In the similar manner, tetra- $O$-acetyl- $\beta$ - $D$-galactosyl isothiocyanate (IIb) (6.2 g), m.p. $92{ }^{\circ} \mathrm{C}$ have been synthesized from tetra- $O$-acetyl- $\alpha-D$-galactosyl bromide (Ib) $(0.024 \mathrm{M}$, $10.0 \mathrm{~g})$ dissolved in sodium dried xylene $(25 \mathrm{~mL})$ in presence of lead thiocyanate $(6.0 \mathrm{~g})$. (Scheme 1). The results were showed in Table 1.

Synthesis of hepta-O-acetyl- $\beta$-D-lactosyl isothiocyanate and hepta-O-acetyl- $\beta$-Dmaltosyl isothiocyanate under microwave irradiation

To a solution of hepta- $O$-acetyl- $\alpha$ - $D$-lactosyl bromide (IV) $(0.014 \mathrm{M}, 10.0 \mathrm{~g})$ dissolved in sodium dried xylene $(25 \mathrm{~mL})$ was added lead thiocyanate $(6.0 \mathrm{~g})$ in a $50 \mathrm{~mL}$ beaker. The reaction mixture was then kept under microwave irradiation for about $40 \mathrm{~min}$. at constant power P-70, the temperature of reaction mixture remain constant at $130-140{ }^{\circ} \mathrm{C}$. The resultant 
solution was then cooled and separated lead bromide was removed by filtration. The solvent was distilled off and sticky mass obtained as residue was triturated with petroleum ether $\left(60-80{ }^{\circ} \mathrm{C}\right)$. A pale yellow solid $(7.2 \mathrm{~g})$ was obtained. The product $(\mathbf{V})$ was purified by chloroform-ether. m.p. $164{ }^{\circ} \mathrm{C}$. (Scheme 2).

In the similar manner, hepta- $O$-acetyl- $\beta$ - $D$-maltosyl isothiocyanate (VIII) $(6.5 \mathrm{~g})$, m.p. $118{ }^{\circ} \mathrm{C}$ have been synthesized from hepta- $O$-acetyl- $\alpha$ - $D$-maltosyl bromide (VII) $(0.014 \mathrm{M}$, $10.0 \mathrm{~g})$ dissolved in sodium dried xylene $(25 \mathrm{~mL})$ in presence of lead thiocyanate $(6.0 \mathrm{~g})$. (Scheme 3). The results were showed in Table 1.

Table 1. Physical data of acetylated sugar isothiocyanates and comparative study of conventional vs. microwave method

\begin{tabular}{|c|c|c|c|c|c|c|c|}
\hline \multirow[b]{2}{*}{ S.No. } & \multirow{2}{*}{$\begin{array}{c}\text { Sugar } \\
\text { isothiocyanates }\end{array}$} & \multirow[b]{2}{*}{$\underset{{ }^{\circ} \mathrm{C}}{\mathrm{m} . \mathrm{p} .}$} & \multicolumn{2}{|c|}{ Conventional method } & \multirow[b]{2}{*}{$\underset{{ }^{\circ} \mathrm{C}}{\text { m.p., }}$} & \multicolumn{2}{|c|}{ Microwave method } \\
\hline & & & $\underset{\mathrm{h}}{\text { Time, }}$ & $\%$ Yield & & $\begin{array}{c}\text { Time, } \\
\text { min }\end{array}$ & $\%$ Yield \\
\hline 1 & IIa & $111-114$ & 3 & 73.83 & 112 & 25 & 89.66 \\
\hline 2 & IIb & $92-94$ & 3 & 58.01 & 92 & 25 & 65.40 \\
\hline 3 & V & $163-165$ & 3 & 63.88 & 164 & 40 & 74.30 \\
\hline 4 & VIII & $118-120$ & 3 & 59.88 & 118 & 40 & 67.07 \\
\hline
\end{tabular}

Synthesis of 1-tetra-O-acetyl- $\beta$-D-glucosyl-3-aryl thiocarbamides and 1-tetra-Oacetyl- $\beta$-D-galactosyl-3-aryl thiocarbamides under microwave irradiation

To benzene solution $(15 \mathrm{~mL})$ of tetra- $O$-acetyl- $\beta$ - $D$-glucosyl isothiocyanate (IIa) $(0.02 \mathrm{M}$, $7.7 \mathrm{~g}$ ) was added appropriate amount of various aryl amines in a $50 \mathrm{~mL}$ beaker. The reaction mixture was then kept under microwave irradiation for about $28 \mathrm{~min}$. at constant power P-70. Afterwards, solvent was distilled off and sticky mass obtained as residue was triturated with petroleum ether $\left(60-80^{\circ} \mathrm{C}\right)$. The product (IIIa) was purified by chloroform-ether. (Scheme 1).

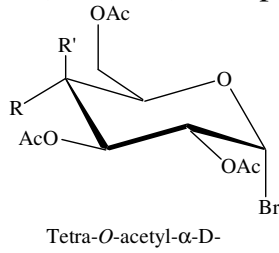

glucosyl/galactosyl bromide

(Ia/Ib)

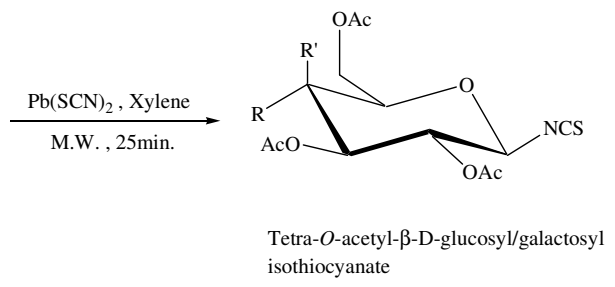

Tetra- $O$-acetyl-
isothiocyanate

(IIa/IIb)
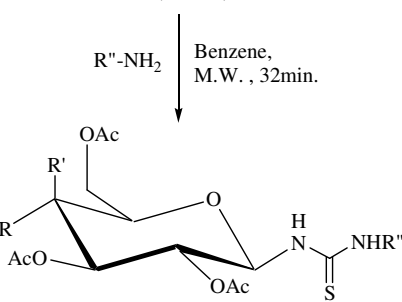

1-Tetra- $O$-acetyl- $\beta$-D-glucosyl/galactosyl

-3-aryl thiocarbamides

(IIIa/IIIb)

Where, i) tetra $-O$-Acetyl- $\beta$ - $D$-glucosyl isothicoyanate; if $\mathrm{R}=\mathrm{OAc}$ and $\mathrm{R}$ ' $=\mathrm{H}$; ii) tetra $-O$-acetyl- $\beta-D$ glucosyl isothicoyanate; if $\mathrm{R}=\mathrm{H}$ and $\mathrm{R}^{\prime}=\mathrm{OAc}$ and iii) $\mathrm{R}^{\prime \prime}=$ a) phenyl, b) $o$-tolyl, c) $m$-tolyl, d) $p$-tolyl, e) $o$-Cl-phenyl, f) $m$-Cl-phenyl, g) $p$-Cl-phenyl

\section{Scheme 1}


In the similar manner, 1-tetra- $O$-acetyl- $\beta$ - $D$-galactosyl-3-aryl thiocarbamides (IIIb) have been synthesized from tetra- $O$-acetyl- $\beta$ - $D$-galactosyl isothiocyanate (IIb) $(0.005 \mathrm{M}$, $2.0 \mathrm{~g}$ ) dissolved in benzene solution with appropriate amount of various aryl amines. (Scheme 1). The results were showed in Table 2.

Table 2. Physical data of acetylated sugar thiocarbamides and comparative study of conventional vs. microwave method

\begin{tabular}{|c|c|c|c|c|c|c|c|c|}
\hline \multirow{2}{*}{$\stackrel{\dot{z}}{\dot{z}}$} & \multicolumn{2}{|c|}{ Compounds } & \multirow{2}{*}{$-{ }^{\circ} \mathrm{C} . \mathrm{C}$} & \multicolumn{2}{|c|}{ Conventional method } & \multirow{2}{*}{$\underset{{ }^{\circ} \mathrm{C} . \mathrm{p}}{\mathrm{m}}$} & \multicolumn{2}{|c|}{$\begin{array}{l}\text { Microwave } \\
\text { method }\end{array}$} \\
\hline & $\begin{array}{c}\text { Sugar } \\
\text { isothiocyanates }\end{array}$ & $\begin{array}{c}\text { Sugar } \\
\text { thiocarbamides }\end{array}$ & & $\begin{array}{c}\text { Time, } \\
\mathrm{h}\end{array}$ & $\begin{array}{c}\% \\
\text { Yield }\end{array}$ & & $\begin{array}{c}\text { Time, } \\
\text { min }\end{array}$ & $\begin{array}{c}\% \\
\text { Yield }\end{array}$ \\
\hline \multirow[t]{7}{*}{1} & IIa & IIIaa & 162 & 3 & 68.42 & 162 & 28 & 74.21 \\
\hline & & IIIab & 170 & 3 & 86.58 & 170 & 28 & 88.36 \\
\hline & & IIIac & 138 & 3 & 81.52 & 138 & 28 & 86.36 \\
\hline & & IIIad & 132 & 3 & 83.08 & 132 & 28 & 84.18 \\
\hline & & IIIae & 160 & 3 & 70.45 & 160 & 28 & 76.50 \\
\hline & & IIIaf & 172 & 3 & 78.27 & 172 & 28 & 80.57 \\
\hline & & IIIag & 145 & 3 & 58.70 & 145 & 28 & 60.78 \\
\hline \multirow[t]{7}{*}{2} & IIb & IIIba & 128 & 3 & 65.58 & 128 & 28 & 70.85 \\
\hline & & IIIbb & 172 & 3 & 54.90 & 172 & 28 & 60.78 \\
\hline & & IIIbc & 160 & 3 & 66.66 & 160 & 28 & 72.54 \\
\hline & & IIIbd & 110 & 3 & 68.23 & 110 & 28 & 74.11 \\
\hline & & IIIbe & 176 & 3 & 71.69 & 176 & 28 & 77.73 \\
\hline & & IIIbf & 124 & 3 & 62.26 & 124 & 28 & 70.18 \\
\hline & & IIIbg & 140 & 3 & 69.81 & 140 & 28 & 76.22 \\
\hline \multirow[t]{7}{*}{3} & V & VIa & 138 & 3 & 82.91 & 138 & 32 & 87.93 \\
\hline & & VIb & 148 & 3 & 79.01 & 148 & 32 & 83.20 \\
\hline & & VIc & 109 & 3 & 76.54 & 109 & 32 & 81.23 \\
\hline & & VId & 124 & 3 & 86.41 & 124 & 32 & 91.35 \\
\hline & & VIe & 149 & 3 & 62.65 & 149 & 32 & 69.87 \\
\hline & & VIf & 153 & 3 & 48.19 & 153 & 32 & 55.42 \\
\hline & & VIg & 139 & 3 & 86.74 & 139 & 32 & 91.56 \\
\hline \multirow[t]{7}{*}{4} & VIII & IXa & 110 & 3 & 75.78 & 110 & 32 & 78.87 \\
\hline & & $\mathrm{IXb}$ & 202 & 3 & 63.56 & 202 & 32 & 65.36 \\
\hline & & IXc & 230 & 3 & 65.79 & 230 & 32 & 67.93 \\
\hline & & IXd & 134 & 3 & 58.00 & 134 & 32 & 61.23 \\
\hline & & IXe & 222 & 3 & 62.18 & 222 & 32 & 64.25 \\
\hline & & IXf & 112 & 3 & 58.00 & 112 & 32 & 60.63 \\
\hline & & IXg & 161 & 3 & 62.88 & 161 & 32 & 65.78 \\
\hline
\end{tabular}

Synthesis of 1-hepta-O-acetyl- $\beta$-D-lactosyl-3-aryl thiocarbamides and 1-hepta-Oacetyl- $\beta$-D-maltosyl-3-aryl thiocarbamides under microwave irradiation

To benzene solution $(15 \mathrm{~mL})$ of hepta- $O$-acetyl- $\beta$ - $D$-lactosyl isothiocyanate $(\mathbf{V})(0.005 \mathrm{M}$, $3.5 \mathrm{~g}$ ) was added appropriate amount of various aryl amines in a $50 \mathrm{~mL}$ beaker. The reaction mixture was then kept under microwave irradiation for about $32 \mathrm{~min}$. at constant power P-70. Afterwards, solvent was distilled off and sticky mass obtained as residue was triturated with petroleum ether $\left(60-80{ }^{\circ} \mathrm{C}\right)$. The product (VIa-g) was purified by chloroform-ether. (Scheme 2). 


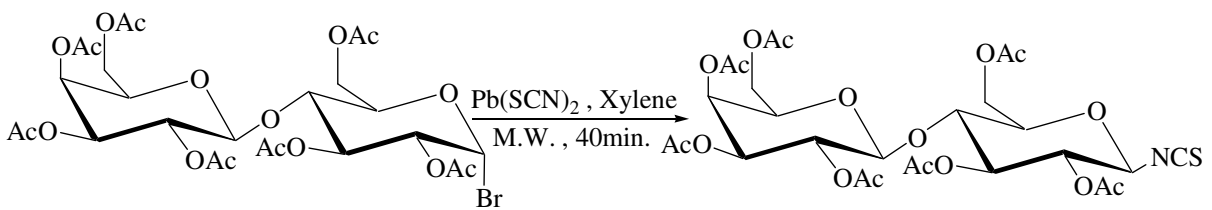

Hepta- $O$-acetyl- $\alpha$-D-lactosyl bromide

(IV)

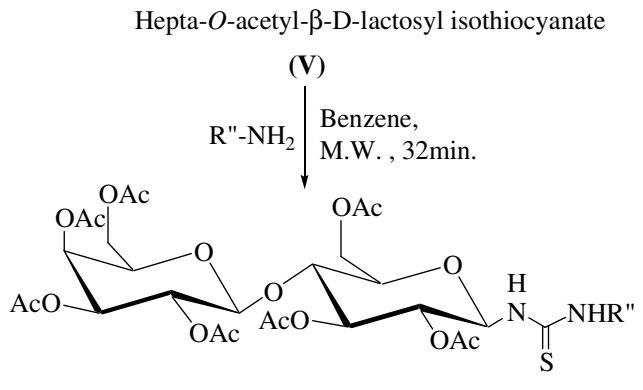

1-Hepta- $O$-acetyl- $\beta$-D-lactosyl-3-aryl thiocarbamides

Where, R" = a) phenyl, b) $o$-tolyl, c) $m$-tolyl, d) $p$-tolyl, e) $o$-Cl-phenyl, f) $m$-Cl-phenyl, g) $p$-Clphenyl.

\section{Scheme 2}

In the similar manner, 1-hepta- $O$-acetyl- $\beta$ - $D$-maltosyl-3-aryl thiocarbamides (IXa-g) have been synthesized from hepta- $O$-acetyl- $\beta$ - $D$-maltosyl isothiocyanate (VIII) $(0.005 \mathrm{M}$, $3.5 \mathrm{~g})$ dissolved in benzene solution with appropriate amount of various aryl amines. (Scheme 3). The results were showed in Table 2.

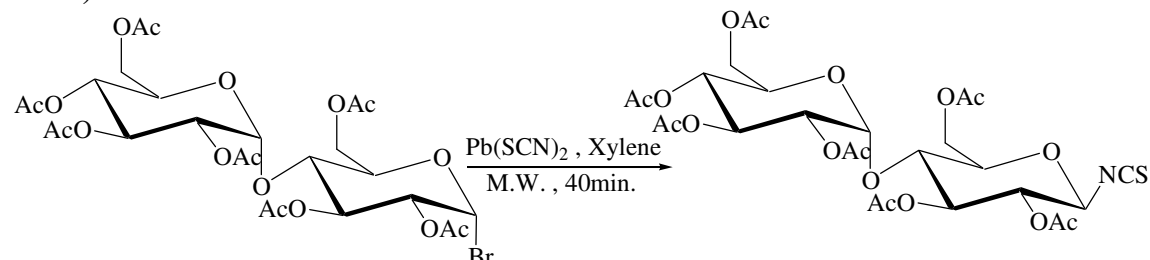

Hepta- $O$-acetyl- $\alpha$-D-maltosyl bromide

(VII)
Hepta- $O$-acetyl- $\beta$-D-maltosyl isothiocyanate

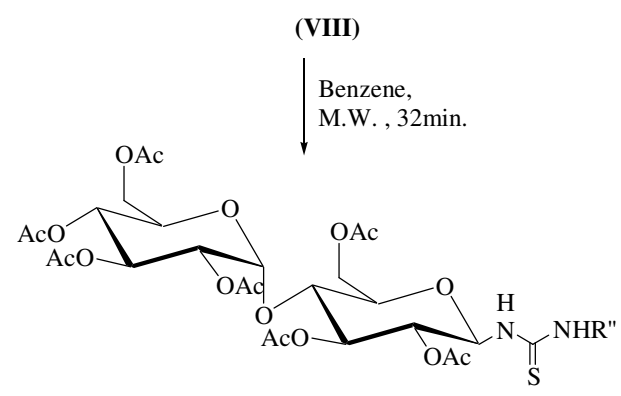

1-Hepta- $O$-acetyl- $\beta$-D-maltosyl-3-aryl thiocarbamides

Where, R" = a) phenyl, b) $o$-tolyl, c) $m$-tolyl, d) $p$-tolyl, e) $o$-Cl-phenyl, f) $m$-Cl-phenyl, g) $p$-Clphenyl.

\section{Scheme 3}




\section{Acknowledgment}

The authors are thankful to UGC, New Delhi for providing financial support for the major research project. [Project File No. 38-208/2009(SR)]. The authors acknowledge the help of SAIF, C.D.R.I., Lucknow. They are also thankful to Principal, Dr. S.G. Bhadange for providing necessary facilities.

\section{References}

1. Corsaro A, Chiacchio U, Pistara V and Romeo G, Curr Org Chem., 2004, 8(6), 511-538.

2. Ubarahande S S, Thakare V G and Berad B N, J Indian Chem Soc., 2010, 87, 1137-1141.

3. Mukerjee A K and Ashare R, Chem Rev., 1991, 91(1), 1-24.

4. Garcia Fernandez J M and Mellet C O, Adv Carbohydr Chem Biochem., 2000, 55, 35-135.

5. Gasch C, Pradera M A, Salameh B A B, Molina J L and Fuentes J, Tetrahedron Asymmetry, 2001, 12, 1267-1277.

6. Fernandez-Bolanos J G, Zafra E, Lopez O, Robina I and Fuentes J, Tetrahedron: Asymmetry, 1999, 10(15), 3011-3023.

7. Todoulou O G, Papadaki-Valiraki A E, Filippatos E C, Ikeda S and De Clercq E, Eur J Med Chem., 1994, 29, 127-131.

8. Povarov L S, Potapova N P, Bakina E V, Preobrazhenskaya M N and Rozynov B V, Bioorg Khim., 1992, 18, 1117-1126.

9. Deshmukh S P, Oriental J Chem., 2000, 16(1), 143-146.

10. Mahalle P R, Korpe G V and Deshmukh S P, J Indian Chem Soc., 2008, 85, 953-958.

11. Tale P V and Deshmukh S P, Heteroatom Chem., 2006, 17(4), 306-309. 


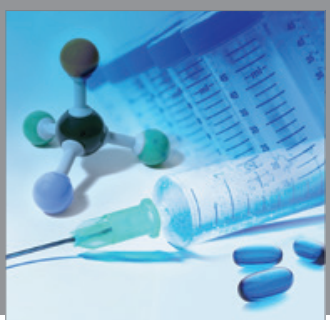

International Journal of

Medicinal Chemistry

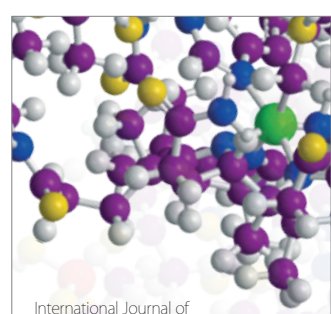

Carbohydrate Chemistry

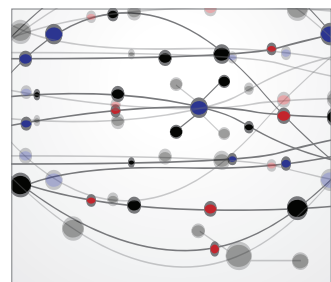

The Scientific World Journal
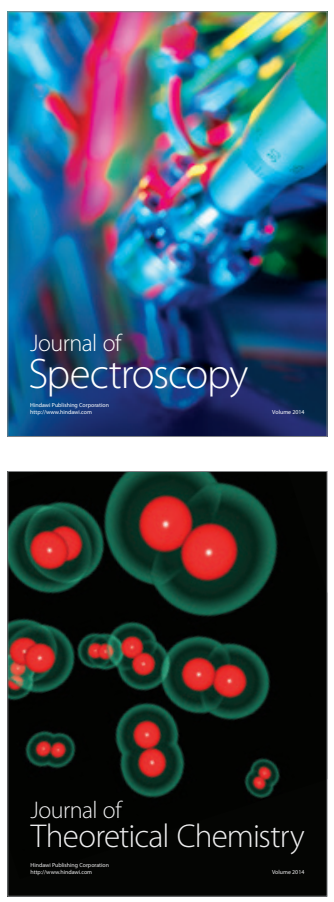
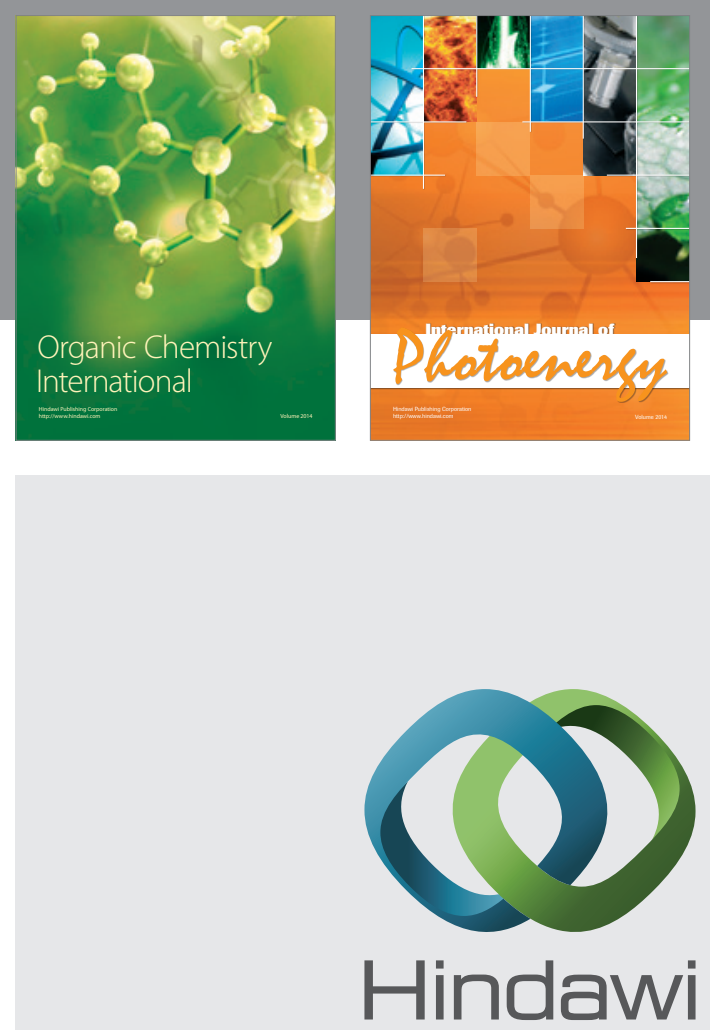

Submit your manuscripts at

http://www.hindawi.com
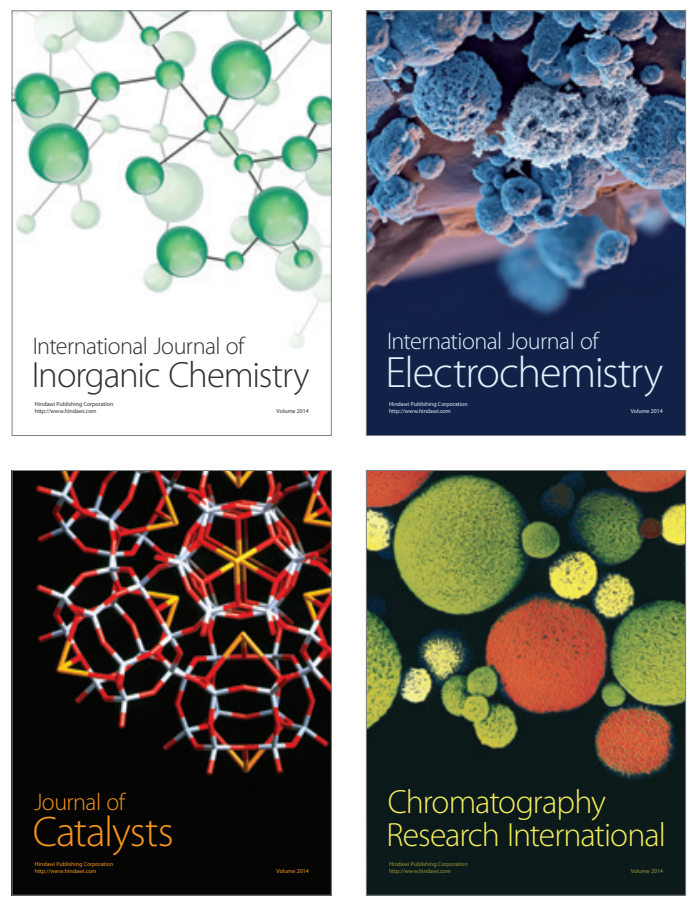
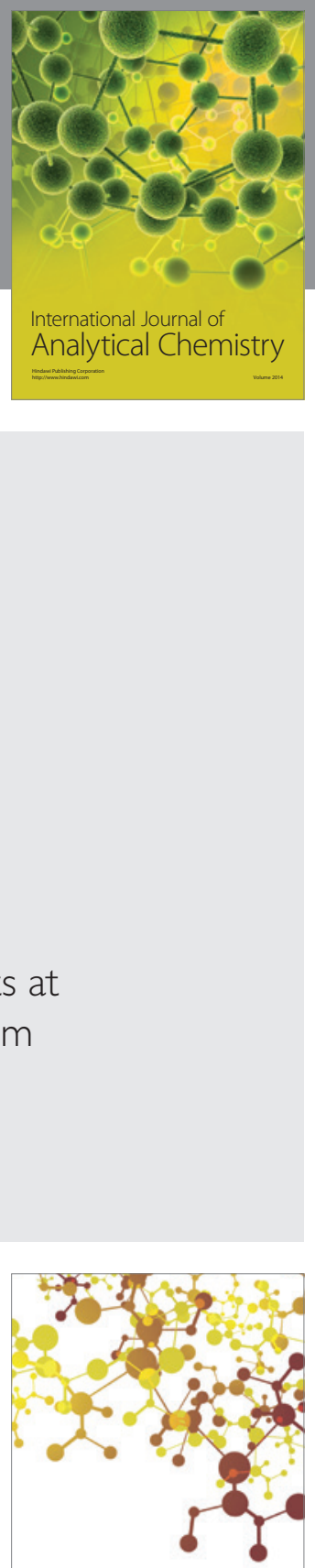

Journal of

Applied Chemistry
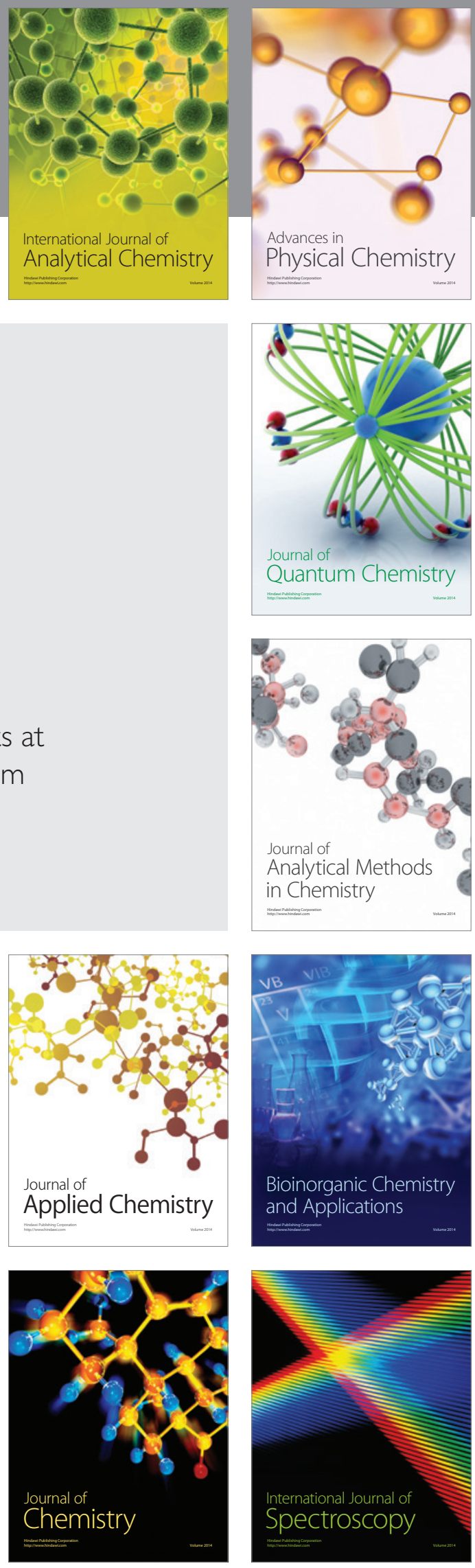